\title{
A COMPARISON OF NEAR INFRARED SPECTRA OF THE GALACTIC CENTER HE I EMISSION LINE SOURCES AND EARLY TYPE MASS LOSING STARS
}

\author{
R. D. BLUM, D. L. DEPOY AND K. SELLGREN \\ The Ohio State University \\ 174 W. 18th Ave., Columbus, Oh, 43210, USA
}

\begin{abstract}
We have obtained $\mathrm{R} \approx 570$ resolution $K$ band spectra of eight sources in the Galactic Center, including four sources within the IRS 16 cluster, IRS 13, IRS 1W, and the compact He I emission line sources AF (also known as $\mathrm{AHH}$ ) and $\mathrm{AHH} \mathrm{NW}$. We have also obtained $\mathrm{R} \approx 570$ $H$ and $K$ band spectra of nine galactic and LMC early-type mass-losing stars, including Ofpe/WN9 and WN stars. The spectra of both the Galactic Center sources and the comparison stars show a wide range of behavior in the He I $(1.70 \mu \mathrm{m}, 2.06 \mu \mathrm{m}, 2.11 \mu \mathrm{m})$ and $\mathrm{H}$ I (Brackett series) lines. We find significantly larger He I equivalent widths in the AF source and two galactic early type mass losing stars than in any of the LMC stars. Several of the Galactic Center He I sources are found to have higher He I velocity widths than any of the galactic or LMC early type mass losing stars. At least one source, IRS 13 , shows a strong red wing to the He I $2.06 \mu \mathrm{m}$ emission.
\end{abstract}

\section{Introduction}

What is the source of radiation that ionizes the gas and powers the emission from dust at the Galactic Center? Two primary models have long been debated: (1) accretion onto a massive black hole and (2) young $\left(\lesssim 10^{7} \mathrm{yr}\right)$ massive stars (see Genzel and Townes 1987 and Genzel, Hollenbach, and Townes 1994 for extensive reviews). Lebofsky, Rieke, \& Tokunaga (1982) identified M supergiant stars in the Galactic Center (GC), supporting the recent star formation model. More recently, Forrest et al. (1987), Allen, 
Hyland, and Hillier (1990) and Krabbe et al. (1991) have identified compact He I sources and/or Br $\alpha$ emission sources with no radio counterparts in the GC. Allen et al. (1990) and Krabbe et al. (1991) interpreted the He I and accompanying $\mathrm{H} \mathrm{I}$ emission as arising in the extended mass-losing envelopes of hot massive post-main-sequence stars. This interpretation is based on the similarity of the $K$ band $(\lambda=2.2 \mu \mathrm{m})$ spectrum of the GC He I source AF and the relative $\mathrm{He} \mathrm{I}$ to $\mathrm{Br} \gamma$ line measurements of other GC He I sources compared to the near infrared (NIR) spectra of optically classified stars in the Large Magellanic Cloud and elsewhere in the Galaxy. In particular, the AF source has been classified as an Ofpe/WN9 star based on its $K$ band spectrum as compared to similar spectra of a number of the LMC stars with this classification (Allen et al. 1990). If correct, this interpretation provides the most substantial evidence to date for the star formation model.

\section{Observations}

The majority of observations were obtained on the nights of 11 and 12 July, 1993 and 6 and 22 September, 1993 on the CTIO $4 \mathrm{~m}$ telescope. The observations were made using the Ohio State Infrared Imager/Spectrometer (OSIRIS) in low resolution mode $(\mathrm{R} \approx 570)$. OSIRIS employs a $256 \times 256$ $\mathrm{HgCdTe}$ array and may be used in crossed-dispersed (simultaneous $I$ (partial), $J, H$ and $K$ ) mode. The GC sources (Figure 1) were observed solely in the $K$ band (except AF) with a $120^{\prime \prime} \times 1.3^{\prime \prime}$ slit. The AF source and comparison stars (Figures 2,3) were observed in cross-dispersed mode with a $20^{\prime \prime} \times 1.3^{\prime \prime}$ slit. The spatial scale was $0.45^{\prime \prime} \mathrm{pix}^{-1}$. A higher resolution $(\mathrm{R} \approx$ 1350) $2.0-2.24 \mu \mathrm{m}$ spectrum of IRS 13 was obtained in July, 1994 on the CTIO $4 \mathrm{~m}$. The spatial scale for this spectrum was $0.17^{\prime \prime} \mathrm{pix}^{-1}$.

\section{Discussion}

The LMC Ofpe/WN9 stars were first classified by Walborn (1977) and they now number ten stars in all (Bohannan and Walborn 1989). These masslosing stars are believed to be precursors to the Wolf-Rayet stage and hence are likely to be helium enriched and massive.

The defining characteristic of the Ofpe/WN9 stars in the NIR has been taken as a He I $2.06 \mu \mathrm{m} / \mathrm{Br} \gamma$ integrated line flux ratio greater than one, with broad line width in He I. Beyond this simple criterion, what more can quantitatively be said regarding the nature of the GC sources in the absence of optical spectra? To begin, we have analyzed the $\mathrm{H} \mathrm{I}$ and $\mathrm{He}$ I emission lines in a number of LMC and galactic (Ofpe/WN9 and other hot supergiant) stars and those in the GC for a direct comparison. A full description of the GC and LMC spectra and their analysis is given in Blum et al. (1994). 


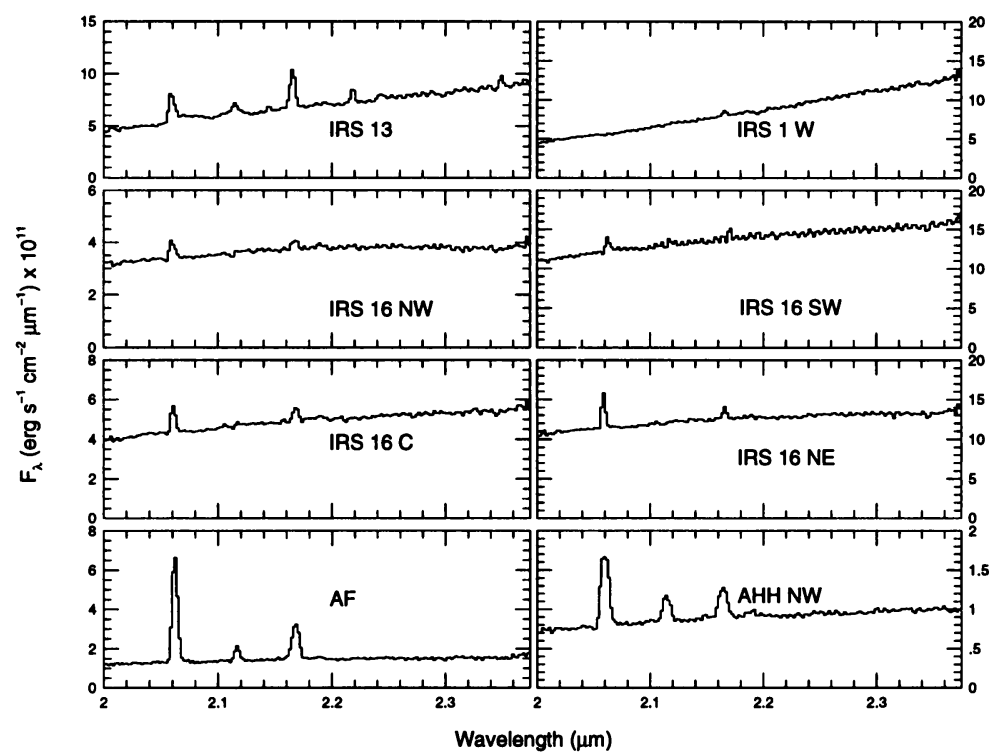

Figure 1. $K$ band spectra of He I emission line sources in the Galactic Center showing the He I $2.06 \mu \mathrm{m}$ and $\operatorname{Br} \gamma(2.17 \mu \mathrm{m})$ emission lines. Several sources also show He I emission at $2.11 \mu \mathrm{m}$. The lines near $2.14 \mu \mathrm{m}, 2.22 \mu \mathrm{m}, 2.24 \mu \mathrm{m}$, and $2.35 \mu \mathrm{m}$ in IRS 13 are due to [Fe III]. These spectra were extracted from $\approx 1.8^{\prime \prime} \times 1.4^{\prime \prime}$ apertures and include background subtraction from nearby $\left(\$^{\prime \prime}\right)$ apertures.

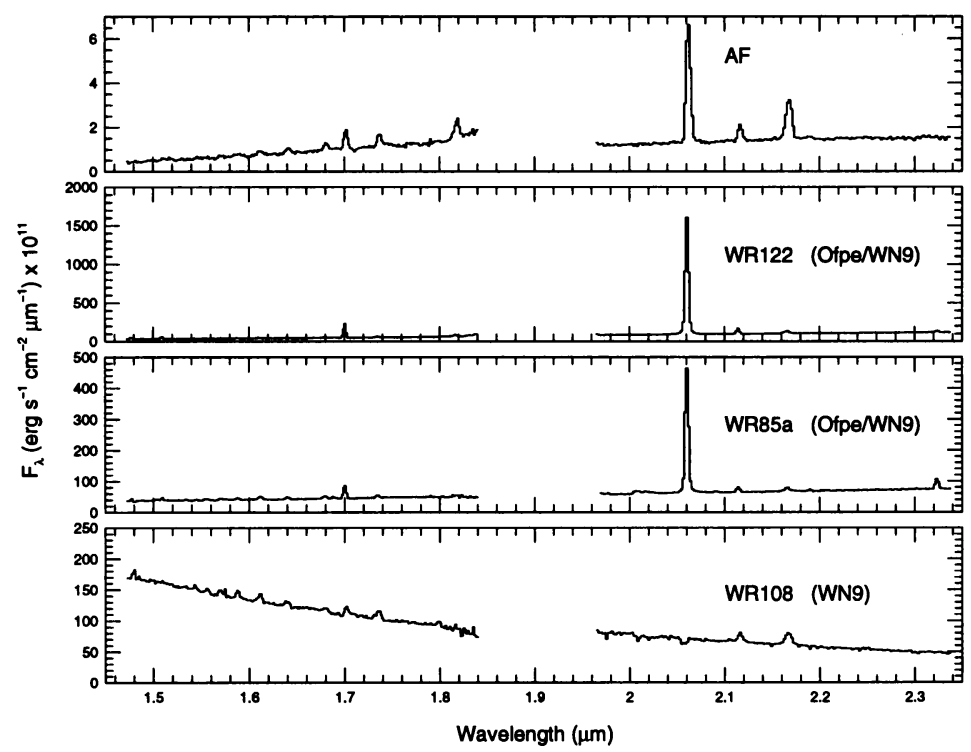

Figure 2. $H$ and $K$ band spectra of He I emission line sources in the Galaxy showing the He I $1.70 \mu \mathrm{m}, 2.06 \mu \mathrm{m}$ and $2.11 \mu \mathrm{m}$ and Brackett series emission lines. Reliable detections are made for $\operatorname{Br} \gamma(2.17 \mu \mathrm{m}), \operatorname{Br} 9(1.82 \mu \mathrm{m}), \operatorname{Br} 10(1.74 \mu \mathrm{m}), \operatorname{Br} 11(1.68$ $\mu \mathrm{m})$, and $\operatorname{Br} 12(1.64 \mu \mathrm{m})$. These spectra were extracted from $\approx 2.3^{\prime \prime} \times 1.4^{\prime \prime}$ apertures and include background subtraction from nearby $\left(\$^{\prime \prime}\right)$ apertures. 


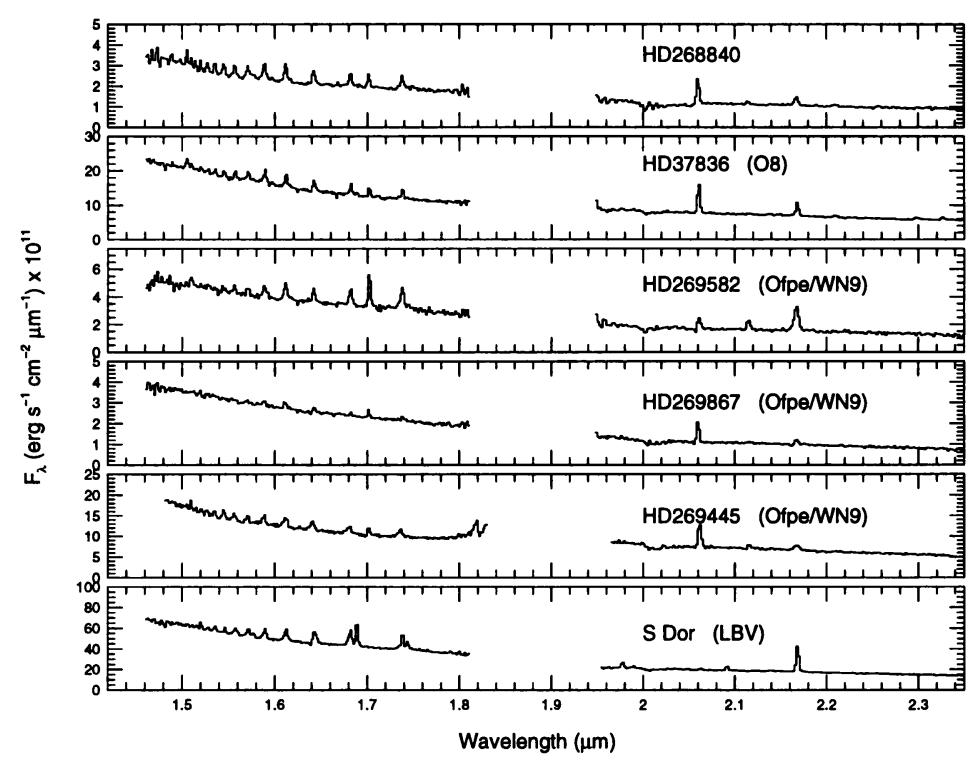

Figure 3. Same as Figure 2 but for He I Emission Line Sources in the LMC. Spectra of the Luminous Blue Variable, S Dor, which shows no He I, is shown for comparison. The lines near $1.69 \mu \mathrm{m}, 1.74 \mu \mathrm{m}$, and $2.09 \mu \mathrm{m}$ are due to Fe II.

The emission line analysis leads to the following results:

1.) The $\mathrm{H} I$ Brackett line ratios with respect to $\mathrm{Br} \gamma$ are larger than predicted for case B recombination (Hummer and Storey $1987 ; \mathrm{T}_{e}=10^{4}{ }^{\circ} \mathrm{K}$, $\mathrm{n}_{e}>10^{4}$ ) for the LMC stars and the galactic star WR85a. This suggests that the lines are optically thick (McGregor, Hyland, and Hillier 1988; Simon et al. 1983). The Brackett lines in the AF source are also interpreted as optically thick, if the extinction to this source is typical of the GC $\left(\mathrm{A}_{K} \approx 3\right.$ mag). The galactic Ofpe/WN9 star WR122 may be consistent with case B or optically thick line ratios depending on the extinction.

2.) The AF, AHH NW, WR85a, and WR122 stars have about an order of magnitude greater He I $2.06 \mu \mathrm{m}$ equivalent width, $\mathrm{W}_{\lambda}$, than the LMC stars. The range of $W_{\lambda}, 109$ to $715 \AA$, for these stars may be compared to the average $\mathrm{W}_{\lambda}=34 \pm 10 \AA$ for the LMC stars. The remainder of the GC sources have much smaller equivalent widths, on average a factor of 2 less than the LMC stars.

3.) All the highest velocity widths are observed in the GC; the galactic and LMC emission-line stars are unresolved at our resolution in the $\mathrm{He} \mathrm{I}$ lines The GC He I widths range from unresolved to $\approx 1200 \mathrm{~km} \mathrm{~s}^{-1}$ (this is the observed FWHM, uncorrected for instrumental broadening).

4.) Several of the GC stars appear to have extended emission to the red of the He I $2.06 \mu \mathrm{m}$ line. IRS 13 (Figure 1) is the most prominent example. The emission may result from blending of unknown lines or may 


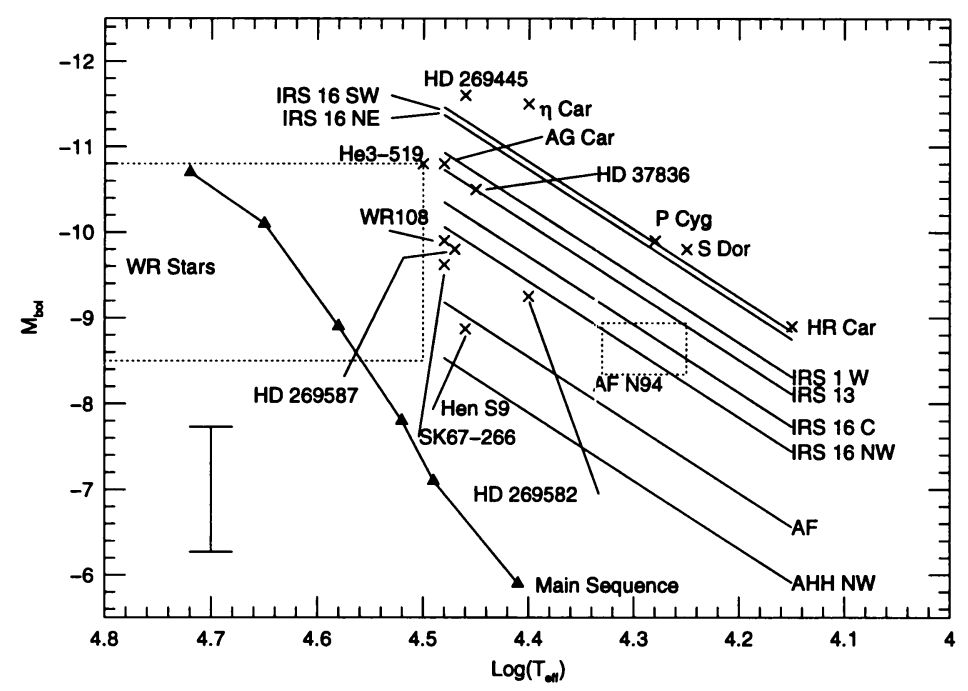

Figure 4. HR diagram for the GC sources and early-type mass-losing stars. The GC sources are shown as solid lines using the bolometric correction $\mathbf{v}$. effective temperature relationship derived in Blum et al. (1994). Data for the individual comparison sources (crosses) are taken from the literature. The larger box (dashed lines) labeled "WR Stars" is reproduced from Humphreys and Davidson (1994). The smaller box (dashed lines) labeled "AF N94" depicts the model results of Najarro et al. (1994) for the AF source. The error bar shown is for the GC sources and corresponds to a \pm 0.71 mag uncertainty in $\mathrm{M}_{\text {bol }}$ estimated from the derived bolometric correction relation added in quadrature to an assumed $\pm 0.5 \mathrm{mag}$ uncertainty in the adopted reddening. The main sequence (Schmidt-Kaler 1982) is shown for comparison (filled triangles).

be associated with the $2.06 \mu \mathrm{m}$ line. A high resolution spectrum of IRS 13 was obtained to further investigate this red wing emission (see Figure 4 of Blum et al. 1994). The wing does not break up into individual lines in the higher resolution spectrum.

5.) Subtraction of nearby background apertures is important in the GC where diffuse gas and unresolved stellar light contaminate the spectra. We find no He I emission in IRS $1 \mathrm{~W}$ after background subtraction (see Figure 1). The embedded source(s) suggested by Rieke and Lebofsky (1982) and Rieke, Rieke, and Paul 1989, in IRS1 W does not appear to be a He I emission-line source. Krabbe et al. (1991) indicate that this source is contaminated by H II emission, but include it as a compact He I source. Libonate et al. (1994) also find no compact He I source at the position of IRS1 W.

Unambiguous spectral types for the GC sources would allow us to place them in the HR diagram and thus, better understand their impact on the GC environment. Our results clearly show significant differences between the LMC stars and the GC He I sources, so we can not yet assign de- 
tailed spectral types; however, their spectra do show broad similarities. Therefore, we have placed the GC stars in the HR diagram (Figure 5) by deriving a bolometric correction at $\mathrm{K}$ from data in the literature and by considering a range of effective temperatures for the GC sources. The details of the construction of the HR diagram may be found in Blum et al. (1994). We consider an upper temperature limit based on temperatures of the Ofpe/WN9 stars and a lower limit based on mass losing stars that show no He I $2.06 \mu \mathrm{m}$ emission. The HR diagram suggests that some of the GC sources may be among the brightest and most massive stars in the Galaxy.

\section{References}

Allen, D. A., Hyland, A. R., \& Hillier, D. J., 1990, MNRAS, 244, 706

Blum, R. D., DePoy, D. L., \& Sellgren, K., 1994, ApJ, submitted

Bohannan, B. \& Walborn, N. R., 1989, PASP, 101, 520

Forrest, W. J., Shure, M. A., Pipher, J. L., \& Woodward, C. E., 1987, in "The Galactic Center," ed. Backer, D. C., AIP, New York

Genzel, R. \& Townes, C. H., 1987, ARAA, 25, 377

Genzel, R., Hollenbach, D. \& Townes, C. H., 1994, "Reports on Progress in Physics, " in press

Hillier, D. J., 1984, ApJ, 280, 744

Hummer, D. G. \& Storey, P. J., 1987, MNRAS, 224, 801

Humphreys, R. M. \& Davidson, K., 1994, PASP, submitted

Krabbe, A., Genzel, R., Drapatz, S., \& Rotaciuc, V., 1991, ApJL, 382, L19

Lebofsky, M. J., Rieke, G. H., \& Tokunaga, A. T., 1982, ApJ, 263, 736

Libonate, S., Pipher, J. L., Forrest, W. J., \& Ashby, M. L. N., 1994, ApJ, in press

McGregor, P. J., Hyland, A. R., \& Hillier D. J., 1988, ApJ, 324, 1071

Najarro, F., Hillier, D. J., Kudritzki, R. P., Krabbe, A., Genzel, R., Lutz, D., Drapatz, S., \& Geballe, T. R., 1994, A\&A, in press.

Rieke, G. H. \& Lebofsky, M. J., 1982, in "The Galactic Center," ed. Riegler \& Blandford, AIP, NY, 194

Rieke, G. H., Rieke, M. J., \& Paul, A. E., 1989, ApJ, 336, 752

Schmidt-Kaler, T., 1982, in Landoldt-Bornstein, Numerical Data and Functional Relationships in Science and Technology, Vol 2, Springer-Verlag, p. 453

Simon, M., Felli, M., Cassar, L., Fischer, J., \& Massi, M., 1983, ApJ, 266, 623

Walborn, N. R., 1977, ApJ, 215, 53

\section{DISCUSSION}

A. Underhill: Have you considered the possibility that the emission lines you are observing may be formed in a disk driven wind? 